\title{
Immune-stimulating Effect of Lactobacillus plantarum Ln1 Isolated from the Traditional Korean Fermented Food, Kimchi
}

\author{
Hye Ji Jang, Hyung-Seok Yu, Na-Kyoung Lee and Hyun-Dong Paik* \\ Department of Food Science and Biotechnology of Animal Resources, Konkuk University, Seoul 05029, Republic of \\ Korea \\ This study aimed to determine the immune-stimulating effects of heat-killed Lactobacillus \\ plantarum $\operatorname{Ln} 1$ (HK-Ln1) through the production of nitric oxide (NO) and pro-inflammatory cytokine \\ achieved by inducing NF-KB and mitogen-activated protein kinase (MAPK)-signaling pathways in \\ macrophages. HK-Ln1 showed higher NO and cytokine production compared to control (non- \\ stimulated lipopolysaccharide); in addition, the expression of inducible nitric oxide synthase (iNOS) \\ was induced through HK-Ln 1 treatment. The phosphorylation of IKB- $a$ and p65 increased following \\ treatment by HK-Ln1, which implicates IKB-a degradation and the translocation of p65 to nucleus. In \\ addition, the phosphorylation of MAPKs, ERK 1/2, JNK, and p38 was induced following HK-Ln1 \\ treatment.
}

Keywords: Lactobacillus plantarum, probiotics, heat-killed cells, immune-stimulating

Received: January 22, 2020 Accepted: March 19, 2020

First published online: March 20, 2020

*Corresponding author Phone: +82-2-2049-6011 Fax: +82-2-455-3082 E-mail:hdpaik@konkuk.ac.kr

pISSN 1017-7825 elSSN 1738-8872

Copyright(C) 2020 by The Korean Society for Microbiology and Biotechnology
Lactic acid bacteria (LAB) are widely known probiotics and para-probiotics used to improve gut condition, barrier function, and immunity [1-4]. LAB is reported to improve the immune system and decrease the risk of infection by bacteria, viruses, and other pathogens [5]. Although the mechanisms of immune system stimulation by LAB has not yet been fully understood, heat-killed probiotics as well as live probiotics are reported to have immune-stimulating effects [6]. Thus, the aim of this study was to evaluate the immune-stimulating effect of heatkilled Lactobacillus plantarum Ln1 (HK-Ln1) isolated from kimchi.

L. plantarum Ln1 isolated from kimchi and L. rhamnosus GG (LGG) were grown in lactobacilli MRS broth (BBL, BD Biosciences, USA) at $37^{\circ} \mathrm{C}$ for $15 \mathrm{~h}$. LGG, used as the control strain, was obtained from Korean Collection for Type Cultures (Korea). To obtain heat-killed Lactobacillus, the cells were heated at $80^{\circ} \mathrm{C}$ for $30 \mathrm{~min}$. The final concentration of heat-killed Lactobacillus was adjusted to $10^{7}$ and $10^{8} \mathrm{CFU} / \mathrm{ml}$, respectively. To investigate the immune-stimulating effects of HK-Ln1, nitric oxide (NO) assay, semi-quantitative real time RCR, and Western blot assay were performed with some modifications $[7,8]$. In addition, we used specific inhibitors such as MAPKs inhibitors (ERK 1/2, PD98059; JNK, SP600125; p38, SB203580) and NF-кB inhibitor (PDTC). $\mathrm{NO}$ is known to be involved in various physiological processes, such as nerve growth, neurotransmission, and regulation of cardiovascular pressure; furthermore, NO has been used for treatment of vascular disorders $[9,10]$. It has been reported that NO plays an important role in immune response and host defense against invading pathogenic bacteria and viruses, as well as tumor cells [11]. NO produced by iNOS plays a physiological role in immune function after LPS stimulation to protect host cells [12]. This study demonstrated immune-stimulatory effect of HK-Ln1 compared to $10 \mathrm{ng} / \mathrm{ml}$ LPS. Generally, in order to evaluate the immune-stimulating other LAB, LPS concentration was performed at $1-10 \mathrm{ng} / \mathrm{ml}$ to minimize the cytotoxic effect $[7,12,13]$. NO production was examined in RAW 264.7 cells by NO assay. HK-Ln1 $\left(10^{8} \mathrm{CFU} / \mathrm{ml}\right)$ showed the highest NO production $(5.31 \mu \mathrm{M})$ compared to that of cell non-treated LPS $(3.74 \mu \mathrm{M})$. NO production was higher in HK-Ln1 $\left(10^{8} \mathrm{CFU} / \mathrm{ml}\right)$ at $5.30 \mu \mathrm{M}$ than in LGG (Fig. 1A). NO assay is convenient tool for detection of immune response. Many studies have reported that probiotics can also stimulate the immune system, resulting in modulation of inflammatory mediators through cytokines that are responsible for the maintenance of the pathological process or immune response in a regulatory sense [14]. In a previous study, NO production of Lactobacillus brevis KCCM 12203P, Lactobacillus paraplantarum SC61, and L. plantarum 200655 was reported to be $21.83,14.77$, and $11.38 \mu \mathrm{M}$, respectively $[7,12,13]$.

When external substances invade the body, macrophages modulate the immune system via production of cytokines such as interleukin (IL)-1 $\beta$, IL-6, and tumor necrosis factor (TNF)- $\alpha$. [12]. In a different study, it was reported that $\mathrm{LAB}$ promotes macrophages through the production of cytokines associated with immune response to foreign materials $[12,15]$. HK-Ln1 showed immunostimulatory effects on the mRNA expression of iNOS, IL- 
A

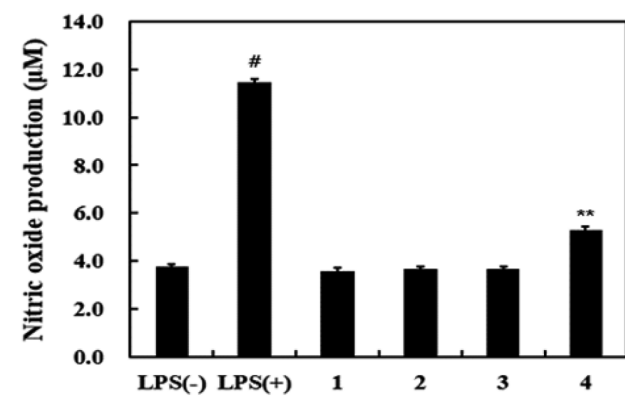

C

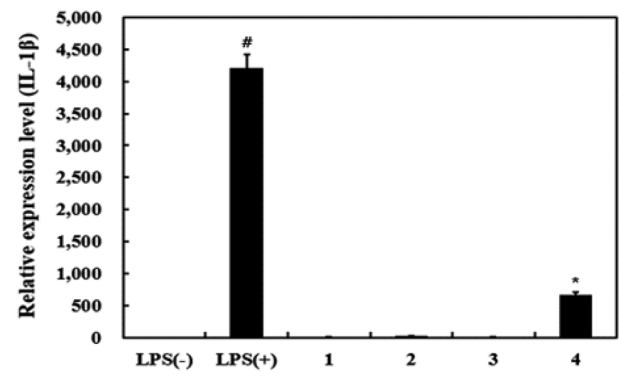

E

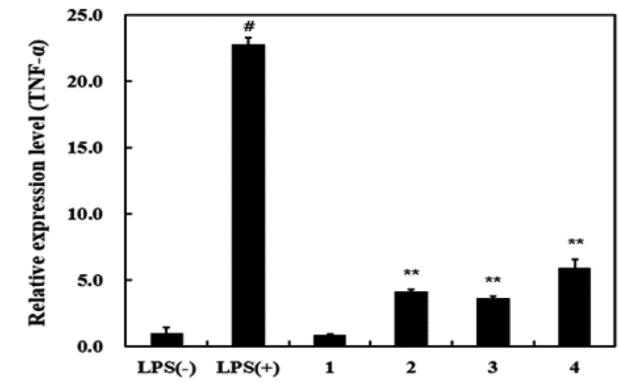

B

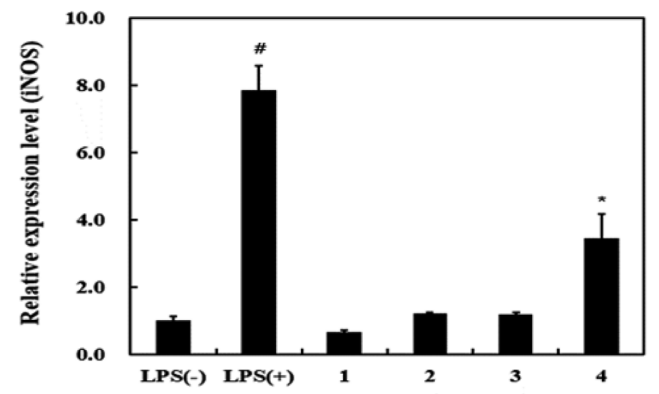

D

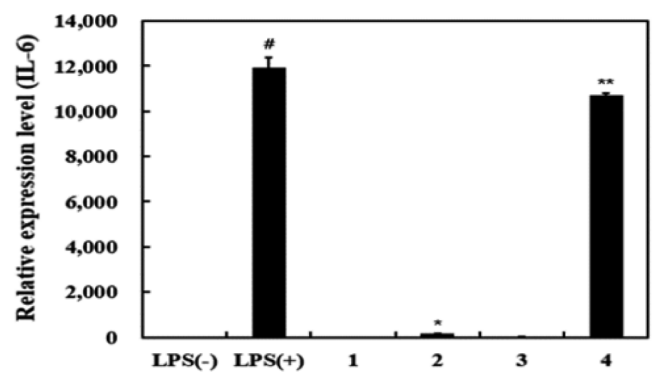

Fig. 1. Effect of nitric oxide production and mRNA expression level of heat-killed L. plantarum Ln1 in RAW 264.7 cells. 1 , L. rhamnosus $\mathrm{GG}\left(10^{7} \mathrm{CFU} / \mathrm{ml}\right) ; 2$, L. plantarum $\mathrm{Ln} 1$ ( $\left.10^{7} \mathrm{CFU} / \mathrm{ml}\right) ; 3$, L. rhamnosus $\mathrm{GG}\left(10^{8} \mathrm{CFU} / \mathrm{ml}\right) ; 4, L$. plantarum Ln 1 ( $\left.10^{8} \mathrm{CFU} / \mathrm{ml}\right)$; LPS (-), non-treated LPS; LPS (+), $10 \mathrm{ng} / \mathrm{ml}$ of treated LPS. (A) Nitric oxide; (B) iNOS; (C) IL-1 $\beta$; (D) IL-6; (E) TNF- $\alpha$. All values are represented as the mean \pm standard deviation of triplicate experiments. $\# p<0.01$, respected to all samples; ${ }^{*} p<0.05,{ }^{* *} p<0.01$, compared to non-treated LPS.

$1 \beta$, IL-6, and TNF- $\alpha$ (Figs. 1B-1E). In this study, to identify immune-stimulating activity of HK-Ln1, we researched by using in vitro anti-inflammatory mechanism through IL- 6 , IL- $1 \beta$, and TNF- $\alpha$, in macrophages or dendritic cells [16]. Many studies reported that Th2 cells produce IL-4, IL-6, IL-10, and TNF- $\alpha$ and regulate humoral immunity [17]. These have shown to stimulate inflammatory response to protect host cells. LPS has known as a direct inducing mediator for producing NO. However, HK-Ln1 is used as a substance that does not induce inflammation but induces immune responses in this study. Previous studies have shown that immunity is enhanced at low LPS levels when inducing inflammation $[7,12,13]$. In comparison with non-treated LPS, the cells cultured with HK-Ln1 $\left(10^{8} \mathrm{CFU} / \mathrm{ml}\right)$ showed higher levels of the following cytokines: iNOS (3.44-fold), IL-1 $\beta$ (669.76-fold), IL-6 (10,697.39-fold), and TNF- $\alpha$ (5.93-fold). The mRNA expression of HK-Ln1 was higher than that of LGG at $10^{7}$ and $10^{8} \mathrm{CFU} / \mathrm{ml}$. L. plantarum 200655 and L. paraplantarum SC61 increased the production of IL-1 $\beta$, IL-6, and TNF- $\alpha[7,12]$. In addition, LAB isolated from kimchi increased the levels pro-inflammatory cytokines including IL-1 $\beta$, IL-6, and TNF- $\alpha$ [18]. Next, we evaluated HK-Ln1 related to iNOS expression (Fig. 2A). Similar to LPS-treatment groups, the protein expression of iNOS was noticeably induced by HK-Ln1 treatment. This result was correlated with up-regulation of NO production and iNOS mRNA expression following treatment by HK-Ln1. The up-regulated pro-inflammatory signature of macrophages led to the hypothesis that HK-Ln1-mediated immune-stimulating effect might be associated with the regulation of NF- $\kappa \mathrm{B}$, a proinflammatory transcriptional factor. The phosphorylation of IкB- $\alpha$ and p65 was increased following treatment with HK-Ln1 which implicates IkB- $\alpha$ degradation and the translocation of p65 to the nucleus (Fig. 2B). Additionally, we verified the NF- $\kappa B$ activation mediated by HK-Ln1 through phosphorylation of I $\kappa B-\alpha$; p 65 was slightly decreased when treated with PDTC, the NF- $\kappa B$-specific inhibitor. NF- $\kappa B$ is known to be a predominant transcriptional factor that modulates pro-inflammatory gene expression. The current results were supported by the previous study demonstrating the translocated NF- $\mathrm{kB}$ induces the expression of pro-inflammatory mediators, including iNOS, COX-2, and cytokines [19, 20].

The MAPKs mediated intracellular signaling pathways contribute to the regulation of cellular functions, such 
A

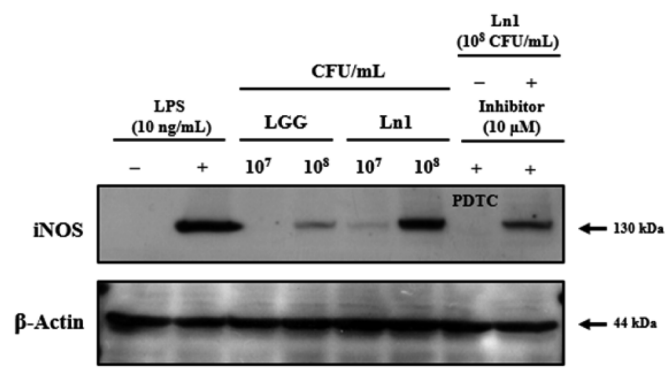

$\mathbf{B}$

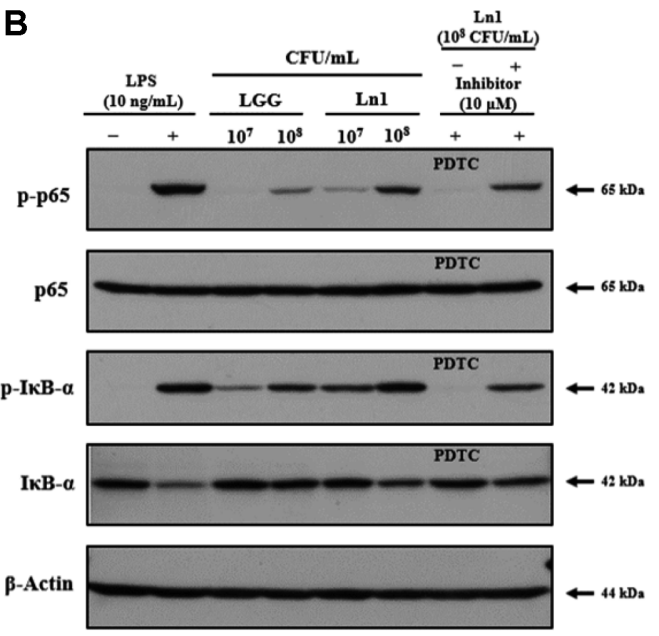

Fig. 2. The effects of heat-killed L. plantarum Ln1 for iNOS expression and for NF-kB activation in RAW 264.7 cells. LGG, L. rhamnosus GG $\left(10^{7}\right.$ and $10^{8} \mathrm{CFU} /$ well $)$; Ln1, L. plantarum $\mathrm{Ln} 1\left(10^{7}\right.$ and $10^{8} \mathrm{CFU} /$ well). All groups were not treated with LPS. LPS (+) was treated with $10 \mathrm{ng} / \mathrm{ml}$. NF-kB inhibitor was used PDTC with $10 \mu \mathrm{M}$. (A) Representative immunoblots of protein expression levels of iNOS and $\beta$-actin. (B) Representative immunoblots of protein expression levels of p-p65, p65, p-ІкB- $\alpha$, IкB- $\alpha$, and $\beta$-actin. Results are representative of three independent experiments.

as gene expression, differentiation, mitosis, apoptosis, and cell survival. MAPKs are crucially involved in transcriptional regulation of pro-inflammatory responses [21, 22]. In particular, p38 MAPKs have been reported to play a vital role in the response to cellular processes [23]. The phosphorylation of MAPKs, ERK 1/2, JNK, and p38, was markedly induced by HK-Ln1 in a dose-dependent manner (Figs. 3A and 3B). The treatment of MAPKs specific inhibitor slightly reduced the HK-Ln1-induced phosphorylation of ERK 1/2, JNK, and p38; furthermore, HK-Ln1 exhibited higher stimulating effect compared to LGG at equal concentrations. Similarly, L. reuteri ATCC PTA 6475 displayed immune-modulating properties in macrophages through the regulation of MAPKs and NF$\kappa \mathrm{B}[24]$. Therefore, these results demonstrated that the immune-stimulating potential of HK-Ln1 was associated with the activation of the NF- $\mathrm{kB}$ and the MAPKs signaling pathway.
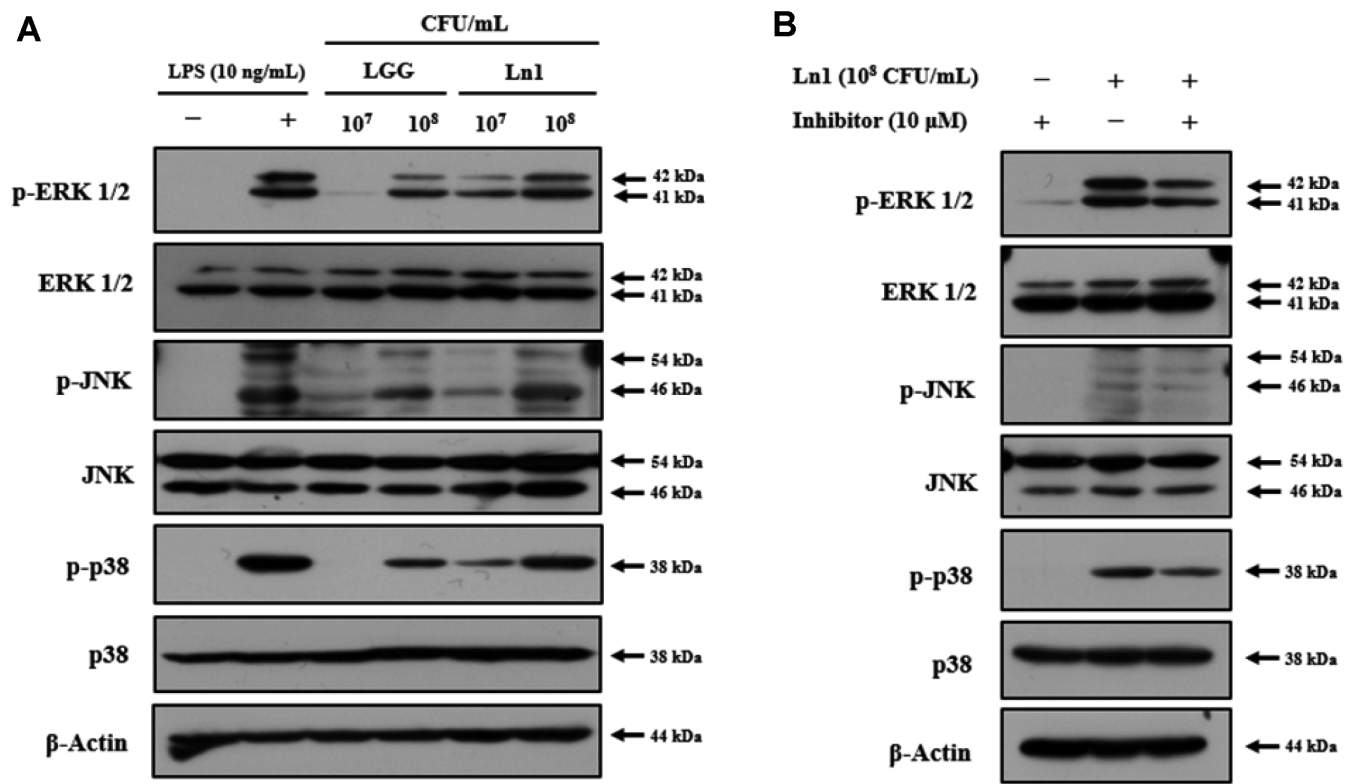

Fig. 3. The effects of heat-killed L. plantarum Ln1 on phosphorylation of MAPKs in RAW 264.7 cells. LGG, $L$. rhamnosus GG $\left(10^{7}\right.$ and $10^{8} \mathrm{CFU} /$ well); Ln1, L. plantarum $\mathrm{Ln} 1$ ( $10^{7}$ and $10^{8} \mathrm{CFU} /$ well). All groups were not treated with LPS. LPS (+) was treated with $10 \mathrm{ng} / \mathrm{ml}$. MAPK inhibitor was used PD98059 (p-ERK 1/2 and ERK 1/2) SP600125 (p-JNK and JNK), and SB203580 (p-p38 and p38) with $10 \mu \mathrm{M}$. (A) Representative immunoblots of protein expression levels of p-ERK1/2, ERK1/ 2, p-JNK, JNK, p-p38, p-38, and $\beta$-actin. (B) Representative immunoblots of protein expression levels of p-ERK1/2, ERK1/2, pJNK, JNK, p-p38, p-38, and $\beta$-actin. Results are representative of three independent experiments. 


\section{Conflict of Interest}

The authors have no financial conflicts of interest to declare.

\section{References}

1. Yang X, Zhou J, Fan L, Qin Z, Chen Q, Zhao L. 2018. Antioxidant properties of a vegetable-fruit beverage fermented with two Lactobacillus plantarum strains. Food Sci. Biotechnol. 27: 1719-1726.

2. Koh WY, Utra U, Ahmad R, Rather IA, Park YH. 2018. Evaluation of probiotic potential and anti-hyperglycemic properties of a novel Lactobacillus strain isolated from water kefir grains. Food Sci. Biotechnol. 27: 1369-1376.

3. Daliri E B, Lee BH, Park BJ, Kim SH, Oh DH. 2018. Antihypertensive peptides from whey proteins fermented by lactic acid bacteria. Food Sci. Biotechnol. 27: 1781-1789.

4. Hassan MT, Jang WJ, Lee BJ, Kim KW, Hur SW, Lim SG, et al. 2019. Heat-killed Bacillus sp. SJ-10 probiotic acts as a growth and humoral innate immunity response enhancer in olive flounder (Paralichthys olivaceus). Fish Shellfish Immunol. 88: 424-431.

5. Fujii T, Jounai K, Horie A, Takahashi H, Suzuki H, Ohshio K, et al. 2017. Effects of heat-killed Lactococcus lactis subsp. lactis JCM 5805 on mucosal and systemic immune parameters, and antiviral reactions to influenza virus in healthy adults; a randomized controlled double-blind study. J. Funct. Foods 35: 513-521.

6. Adams CA. 2010. The probiotic paradox: Live and dead cells are biological response modifiers. Nutr. Res. Rev. 23: 37-46.

7. Yang SJ, Lee JE, Lim SM, Kim YJ, Lee NK, Paik HD. 2019. Antioxidant and immune-enhancing effects of probiotic Lactobacillus plantarum 200655 isolated from kimchi. Food Sci. Biotechnol. 28: 491-499.

8. Chang CK, Wang SC, Chiu CK, Chen S Y, Chen ZT, Duh PD. 2015. Effect of lactic acid bacteria isolated from fermented mustard on immune potentiating activity. Asian Pac. J. Prop. Biomed. 5: 281-286.

9. MacMicking J, Xie QW, Nathan C. 1997. Nitric oxide and macrophage function. Annu. Rev. Immunol. 15: 323-350.

10. Bogdan C. 2001. Nitric oxide and the immune response. Nat. Immunol. 2: 907-916.

11. Ahmad W, Jantan I, Kumolosasi E, Haque MA, Bukharu SNA. 2018. Immunomodulatory effects of Tinospora crispa extract and its major compounds on the immune functions of RAW 264.7 macrophages. Int. Immunophamacol. 60: 141-151.

12. Song MW, Jang HJ, Kim KT, Paik HD. 2019. Probiotic and antioxidant properties of novel Lactobacillus breivs KCCM 12203P isolated from kimchi and evaluation of immune-stimulating activities of its heat-killed cells in RAW 264.7 cells. J. Microbiol. Biotechnol. 29: 1894-1903.

13. Son SH, Yang SJ, Jeon HL, Yu HS, Lee NK, Park YS, et al. 2018. Antioxidant and immunostimulatory effect of potential probiotic Lactobacillus paraplantarum SC61 isolated from Korean traditional fermented food, jangajji. Microb. Pathog. 125: 486-492.

14. Ferreira dos santos T, Melo TA, Almeida ME, Rezende RP, Romano CC. 2016. Immunomodulatory effects of Lactobacillus plantarum Lp62 on intestinal epithelial and mononuclear cells. Biomed. Res. Int. 2016: 8404156.

15. Choi HJ, Lee NH, Paik HD. 2015. Health benefits of lactic acid bacteria isolated from kimchi, with respect to immunomodulatory effects. Food Sci. Biotechnol. 24: 783-789.

16. Devi SM, Kurrey NK, Halami PM. 2018. In vitro anti-inflammatory activity among probiotic Lactobacillus species isolated from fermented foods. J. Funct. Foods 47: 19-27.

17. Choi M, Lee Y, Lee NK, Bae CH, Park DC, Paik HD, et al. 2019. Immunomodulatory effects by Bifidobacterium longum KACC 91563 in mouse splenocytes and macrophages. J. Microbiol. Biotechnol. 29: 1739-1744.

18. Hur HJ, Lee KW, Lee HJ. 2004. Production of nitric oxide, tumor necrosis factor- $\alpha$ and interleukin-6 by RAW cell 264.7 macrophage cells treated with lactic acid bacteria isolated from kimchi. Biofactors 21: 123-125.

19. Li C, Meng M, Chen D, Wang Z, Han H, Chen H, et al. 2013. The immunostimulatory effect of novel immunostimulator CH2b with a thiazolidin-4-one ring on the functions of LPS-activated RAW 264.7 macrophages in vitro. Int. Immunopharmacol. 17: 698-703.

20. Tak PP, Firestein GS. 2001. NF-кB: A key role in inflammatory diseases. J. Clin. Invest. 107: 7-11.

21. Kim KN, Heo SJ, Yoon WJ, Kang SM, Ahn G, Yi TH, et al. 2010. Fucoxanthin inhibits the inflammatory response by suppressing the activation of NF- $\mathrm{kB}$ and MAPKs in lipopolysaccharide-induced RAW 264.7 macrophages. Eur. J. Pharmacol. 649: 369-375.

22. Pearson G, Robinson F, Beers Gibson T, Xu BE, Karandikar M, Berman K, et al. 2001. Mitogen-activated protein (MAP) kinase pathways: regulation and physiological functions. Endocr. Rev. 22: 153-183.

23. Zhang S, Yu J, Wang H, Liu B, Yue X. 2019.p38 MAPK is involved in the immune response to pathogenic Vibrio in the clam Meretrix petechialis. Fish Shellfish Immunol. 95: 456-463.

24. Thomas CM, Versalovic J. 2010. Probiotics-host communication. Gut Microbes 3: 148-163. 